Boletín de la Sociedad Geológica Mexicana

VOLUMEN 63, NÚM. 3, 2011, P. 421-427

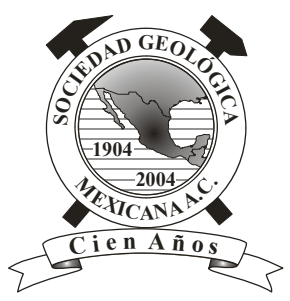

\title{
Las colecciones científicas y la protección del patrimonio paleontológico
}

\author{
Alejandro Cristín ${ }^{1, *} ;$ María del Carmen Perrilliat ${ }^{2}$ \\ ${ }^{1}$ Posgrado en Ciencias de la Tierra, Instituto de Geología, Universidad Nacional Autónoma de México, Ciudad Universitaria, 04510 , \\ México, D. F., México \\ ${ }^{2}$ Instituto de Geología, Universidad Nacional Autónoma de México, Ciudad Universitaria, 04510, México, D. F., México \\ *alcris@unam.mx
}

\section{Resumen}

La función e importancia de las colecciones científicas radica en los objetos de naturaleza única que contienen, materia prima para generar y validar conocimiento científico. Las colecciones paleontológicas preservan, conservan y mantienen disponible los fósiles y su información. Con ello, protegen el patrimonio más preciado de la sociedad: el conocimiento científico de al menos una parte de la naturaleza y sus beneficios sociales.

Palabras clave: Colecciones científicas, colecciones paleontológicas, fósiles.

\begin{abstract}
The function and importance of scientific collections lies in the objects of unique nature that they contain, the raw material to generate and validate scientific knowledge. The paleontological collections preserve, maintain, and keep available fossils and the information they contain. In this way they protect the most valuable heritage of society: the scientific knowledge of at least a part of nature and its social benefits.
\end{abstract}

Keywords: Scientific collections, paleontological collections, fossils.

\section{Introducción}

La República Mexicana posee una gran riqueza natural, y su conocimiento, uso racional, conservación y protección debe ser una prioridad fundamental para garantizar el desarrollo del país. Las colecciones científicas son entidades imprescindibles para esta labor, puesto que en ellas se construye el entendimiento científico de dicha riqueza y en el proceso se derivan, tarde o temprano, numerosos beneficios sociales.

Si bien todas las colecciones científicas buscan perpetuamente presupuesto, infraestructura, personal capacitado y respeto por parte de usuarios, curadores y de personas encargadas de la administración institucional; ésta situación es especialmente difícil para las colecciones paleontológicas mexicanas, consecuencia de la ignorancia que existe sobre su papel y relevancia.

El presente artículo ofrece un panorama sobre la naturaleza de cualquier colección científica, su función e importancia. En este contexto se puntualiza la relevancia de las colecciones paleontológicas en el desarrollo de la Paleontología en México. Con ello se pretende dar respuesta a la necesidad de difundir la siguiente idea: las colecciones científicas son una condición necesaria para la existencia 
de la paleontología en México.

\section{Colecciones científicas}

Las colecciones científicas tienen como característica común el concentrar archivos del conocimiento en un espacio determinado con el fin de conservarlos y mantenerlos disponibles en el largo plazo. Estos archivos tienen la cualidad de ser objetos físicos, denominados ejemplares, que son de suma importancia para la ciencia. Los ejemplares son de naturaleza muy diversa, por ejemplo: polen, semillas, plantas prensadas, hongos secos, conchas micro y macroscópicas, exoesqueletos de insectos, huevos de ave, pieles y esqueletos de mamíferos; minerales, núcleos de hielo, rocas, fósiles, meteoritos; puntas de flecha, cuchillos de piedra, vasijas, estatuillas, incensarios, máscaras; utensilios étnicos como canastas, zapatos, brazaletes, muñecas, tapetes, canoas; pinturas y otras obras de arte; fotografías, cintas magnéticas de video o audio; artefactos y máquinas como computadoras, trajes de astronauta, barcos de guerra, aviones, satélites, naves espaciales, entre otros (Figura 1).

Entender la función y la importancia de cualquier colección científica necesariamente implica entender primero una propiedad de los ejemplares: su naturaleza única y por otra parte, la relación que tienen con la generación y avance del conocimiento científico.

La naturaleza única de los ejemplares se debe a que los procesos involucrados en su creación son dependientes del

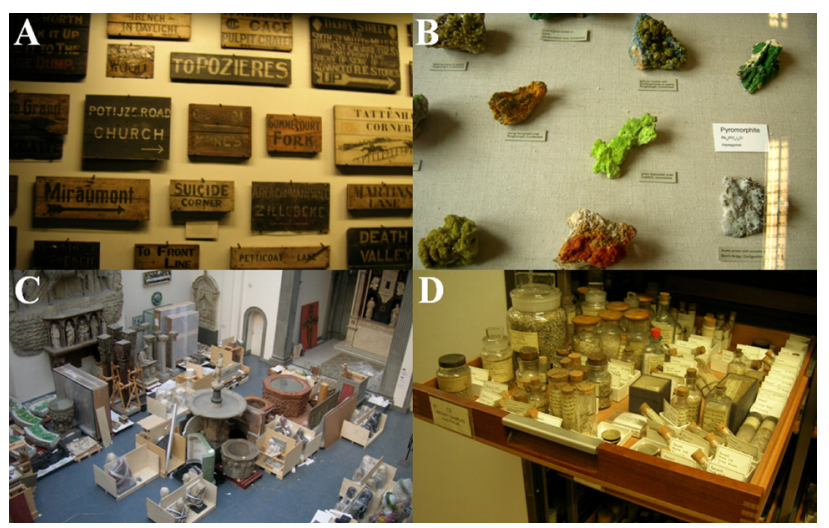

Figura 1. Colecciones Científicas y su exhibición. Una función complementaria de varias instituciones que albergan colecciones científicas es la exhibición de una parte del acervo. Con ello se pretende difundir y divulgar el conocimiento científico así como deleitar a todo tipo de público a través de exposiciones permanentes y temporales. Las siguientes imágenes ejemplifican los tipos de colecciones científicas y de exhibición. A) Colección Histórica, exhibición permanente de letreros que identificaban las trincheras de la Primera Guerra Mundial, Imperial War Museum, Londres. B) Colección Mineralógica en exhibición permanente, Natural History Museum, Londres. C) Colección de Arte en proceso de empaque para el transporte y montaje de una exhibición temporal, Victoria and Albert Museum, Londres. D) Colección Micropaleontológica, acervo exclusivo para investigación, Natural History Museum, Londres. Fotogafías: A. Cristín tiempo y del azar, siendo irrepetibles. Es por ello que las colecciones científicas concentran y preservan ejemplares e información relacionada con el contexto en el cual éstos son hallados y recolectados. El contexto puede ser de diversa naturaleza, por ejemplo ecológico, geológico, arqueológico, geográfico, climático, etc.

A partir de los ejemplares y su información es posible el estudio científico de lo que Gould (1989) denominó sistemas históricos (procesos sociales, biológicos, geológicos cosmológicos, etc.), pues las interpretaciones y sus evidencias (los ejemplares) pueden ser puestas al escrutinio de la comunidad científica. Las colecciones científicas permiten generar, validar o perfeccionar el conocimiento científico. Cotterill (1997) defiende este papel relevante de las colecciones apoyándose en el falsacionismo de Karl Popper. El autor argumenta que las colecciones preservan los ejemplares e información, y los mantiene disponibles a la comunidad científica. Con ello se asegura el poder poner a prueba (intentar falsear) cualquier hipótesis o interpretación. Sin la existencia de las colecciones, no existiría la oportunidad de validar la información, las hipótesis o interpretaciones derivadas del estudio de los ejemplares.

Se puede modificar el argumento de Cotterill (1997) con la sustitución de otro esquema filosófico y verificar que la importancia de las colecciones científicas es indiscutible. Siguiendo a Khun (1962), los ejemplares constituyen evidencias que soportan paradigmas y periodos de ciencia normal, o son evidencias anómalas que con el paso del tiempo contribuyen a detonar revoluciones científicas.

Las colecciones científicas pueden ser clasificadas en función de las ciencias con las que se relacionan y de la naturaleza de objetos que concentran (Tabla 1). En general, las colecciones de historia natural contienen los ejemplares asociados al desarrollo histórico, social y cultural del hombre (Tate y Skinner, 1992; Gowland, 1997); mientras que las colecciones de historia natural son aquellas que contienen los registros físicos de las formas de vida y de los procesos terrestres (Brunton et al., 1985; Standsfield, 1994; Nudds y Pettitt, 1997). Por convención, éstas últimas se han agrupado principalmente en colecciones biológicas, geológicas y paleontológicas, cada una con características propias.

Las colecciones son alojadas en museos, sitios especializados dedicados al resguardo, mantenimiento y administración de dicho material científico. El Artículo 3 del Estatuto vigente del Consejo Internacional de Museos (ICOM, 2007a; ICOM por sus siglas en inglés), define al museo como "[...] una institución permanente, sin fines de lucro, al servicio de la sociedad y su desarrollo, abierta al público y que realiza investigaciones referidas a los testigos materiales del hombre y su entorno, los adquiere, conserva, divulga y, en particular, los expone para fines de estudio, educación y deleite". En el Artículo 4 (ICOM, 2007b) e integrado a esta definición, el ICOM admite además de los museos propiamente dichos, a: 
Tabla 1. Tipos de colecciones científicas. Con base en un criterio antropológico existen dos grandes grupos de colecciones científicas: historia social (a) e historia natural (b). La gran variedad de colecciones biológicas (en b) se debe a la naturaleza diversa de los ejemplares, los requerimientos para su manejo y almacenamiento, y a una fuerte tradición histórica.

\begin{tabular}{|c|c|c|c|c|}
\hline & & Colecciones arqueológicas & & \\
\hline & & Colecciones antropológicas & & \\
\hline & (o) Hi-tonio oniol & Colecciones etnográficas & & \\
\hline & (a) Hitsiorila soutal & Colecciones de arte & & \\
\hline & & Filmotecas & & \\
\hline & & Audiotecas & & \\
\hline & & & & Algas \\
\hline & & & Botánicas y herbarios & Hongos \\
\hline & & & & Plantas \\
\hline & & & & $\begin{array}{l}\text { Invertebrados } \\
\text { marinos }\end{array}$ \\
\hline & & & Invertebrados & $\begin{array}{l}\text { Invertebrados } \\
\text { terrestres }\end{array}$ \\
\hline & & & & Entomológicas \\
\hline & & Colecciones hiclóqicos & & Peces \\
\hline Colecciones científicas & & Colectionies oiologicas & Vertebrados & $\begin{array}{l}\text { Anfibios y } \\
\text { reptiles }\end{array}$ \\
\hline & & & & Mamíferos \\
\hline & & & & Ceparios \\
\hline & (b) Historia Natural & & & $\begin{array}{l}\text { Jardines } \\
\text { botánicos }\end{array}$ \\
\hline & & & Colecciones vivas & Herpetarios \\
\hline & & & & Acuarios \\
\hline & & & & Zoológicos \\
\hline & & & Mineralógicas & \\
\hline & & Colecciones geológicas & Meteoritos y materiale & lanetarios \\
\hline & & & Litológicas & \\
\hline & & & Núcleos (roca, hielo, s & \\
\hline & & & Micropaleontológicas & \\
\hline & & 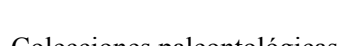 & Paleobotánicas & \\
\hline & & Coicecionies parevilutiogicas & Invertebrados fósiles & \\
\hline & & & Vertebrados fósiles & \\
\hline
\end{tabular}

a) Los institutos de conservación y galerías de exhibición que dependen de las bibliotecas y centros de archivo; b) Los sitios y monumentos arqueológicos, etnográficos y naturales así como los sitios y monumentos históricos que por sus actividades de adquisición, conservación y comunicación tengan la naturaleza de museo;

c) Las instituciones que exhiben especímenes vivientes como los jardines botánicos y zoológicos, acuarios, viveros, etc.;

d) Las reservas naturales;

e) Los centros científicos y planetarios.

Así, el concepto de museo es mucho más amplio de lo que tradicionalmente se piensa, y con éste se abandona la idea de independencia o falta de vínculo entre las colecciones y sus instituciones hospederas. Las colecciones constituyen una parte, si no es que la sine qua non de las instituciones hospederas, por lo que todo aquello que afecta a uno afecta al otro. Desafortunadamente este es un axioma poco comprendido con importantes repercusiones.

Las colecciones y los museos tienen una gran responsabilidad social, sobre todo porque la mayoría dependen económicamente de los contribuyentes. Mucho se ha discutido de su función social (Nudds y Pettitt, 1997; Allmon, 2005) y como consecuencia se ha reconocido su valor monetario, educativo, público, cultural e histórico (Allmon, 1994; Fitzgerald et al., 1997; Miller et al., 
2004; Suarez y Tsutsui, 2004). Sin embargo, la principal responsabilidad social de los museos es procurar la existencia de sus colecciones, trascendentales para el entendimiento científico de la naturaleza, generador de la riqueza material y el bienestar de toda sociedad (Krikken, 1997; Gánem, 2003).

En el ámbito de las colecciones que nos competen, las de historia natural, la asociación Natural Science Collections Alliance listó algunos de los estudios con beneficios sociales más relevantes (Tobin y Evans, 2004):

1. Entendimiento del clima. Numerosos ejemplares (minerales, rocas, exoesqueletos de invertebrados, plantas, etc.) funcionan como registros o dosímetros naturales de las condiciones climáticas en un momento determinado (cantidad de lluvia, periodos de secas, concentración de gases o temperatura).

2. Entendimiento y protección de la biodiversidad. Los ejemplares y sus respectivos registros de colecta permiten predecir la reacción que tienen las especies a los cambios que sufren los ecosistemas, consecuencia del cambio climático.

3. Biomedicina. La investigación biomédica tiene fundamento en el conocimiento de la materia prima para la obtención de compuestos bioquímicos. $\mathrm{La}$ mayoría son obtenidos de plantas y animales, y las colecciones permiten la identificación taxonómica precisa, así como información sobre el hábitat o las condiciones de crecimiento apropiadas.

4. Extinciones. Dos aspectos mínimos para entender los procesos de extinción son el conocer con certeza aquellas especies ya extintas, y por otra parte, aquellas que se encuentran en peligro de extinción. Esto es posible con las colecciones, pues con el acervo de ejemplares e información se pueden reconocer patrones, cuantificar la intensidad de las extinciones y conservar especies en riesgo.

5. Rastreo de pestes y plagas. De manera semejante, las colecciones constituyen un acervo útil y necesario para la identificación, rastreo y monitoreo de pestes y plagas. Las colecciones ofrecen la ventaja de identificar y comparar especies vectoras y recuperar información ecológica, todo de forma rápida y confiable, aspecto de gran relevancia debido a las consecuencias negativas que tienen las pestes y plagas en economía y salud pública.

6. Manejo de recursos naturales. La exploración y explotación de los recursos tiene un fuerte fundamento en observaciones de campo, así como en el estudio de muestras mineralógicas, geológicas o biológicas. Esta actividad se realiza diariamente en el mundo, por agencias y oficinas gubernamentales o por empresas privadas. Debido a que las colecciones concentran las evidencias físicas de todos los recursos naturales de un país, el impacto ambiental y los gastos de la exploración se ven reducidos. Por otra parte los gobiernos cuentan con el conocimiento científico para el manejo apropiado y la administración eficiente de los recursos.

Con base en lo expuesto, no es trivial señalar que la comunidad científica mexicana tiene la obligación de difundir y divulgar la importancia de las colecciones científicas. También tiene la obligación de promover la creación responsable de colecciones y de proteger a las existentes. Las colecciones científicas y museos deben ser entendidos como lo son en otras naciones: un recurso imprescindible para el desarrollo del país (Cranbrook, 1997; Uribe, 1997; Dalton, 2003; NSTC, CS y IWGSC, 2009).

\section{Colecciones paleontológicas}

La paleontología no sería una ciencia sin la existencia de colecciones paleontológicas. Cada fósil es una fuente de información única e irremplazable con los cuales se hacen interpretaciones geológicas, sistemáticas, paleobiológicas, paleobiogeográfricas, evolutivas y paleoclimáticas (Figura 2). Sin su existencia física o su falta de disponibilidad, todo lo que se escriba o diga de ellos es simplemente un escenario.

Así, la misión de las colecciones paleontológicas es "[...] proporcionar la documentación física de la investigación paleontológica mediante la disponibilidad, a través de la curación, de una muestra representativa y la información asociada de cada taxón en la historia de la vida" (Allmon, 2000).

Es importante hacer énfasis en un concepto clave: la curación. Se entiende por curación a un conjunto de numerosas actividades y diversos procedimientos que

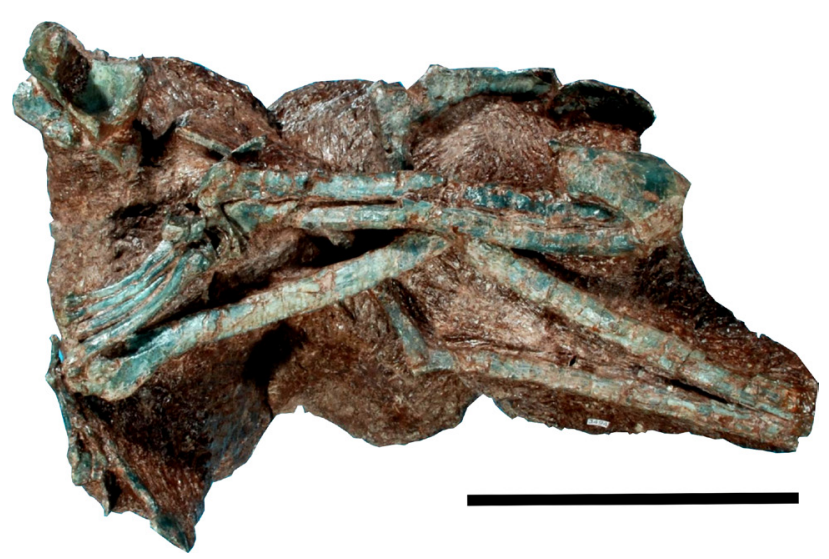

Figura 2. Dimorphodon weintraubi Clark, Hopson, Hernández, Fastovsky y Montellano, 1998. Este esqueleto fósil de un pterodáctilo fue hallado en la Formación La Boca (Jurásico Temprano), Tamaulipas, México. El ejemplar está albergado en la Colección Nacional de Paleontología del Instituto de Geología, UNAM (ejemplar IGM 3494) y sirvió para que Clark et al. (1998) explicaran el modo con el cual los pterodáctilos primitivos se desplazaban. Los autores descartaron un bipedalismo rápido, reinterpretando un desplazamiento lento de hábitos trepadores. Escala 10 $\mathrm{cm}$. Fotografía: J. M. Contreras 
buscan mantener la utilidad y accesibilidad de los ejemplares e información al corto, mediano y largo plazo; por otra parte, con la curación se mantiene una organización eficiente de la información y del acervo (Tabla 2).

Si bien esta definición podría resultar incompleta pues la actividad curatorial trasciende las fronteras de otras actividades como las administrativas, gerenciales, académicas, museográficas e incluso de investigación, ésta es lo suficientemente apropiada para afirmar que sin la curación las colecciones no existirían.

El carácter científico de una colección está determinado principalmente por la naturaleza de los ejemplares que alberga, la información que contiene y el servicio que ofrece a la comunidad científica; sin embargo, también es válido afirmar que está determinado por la curación. Resulta difícil visualizarlo así porque tradicionalmente la curación es una actividad poco reconocida y a su vez desdeñada, que consume mucho tiempo y dinero y carece de resultados espectaculares; pero la curación es tan importante como la investigación científica.

Desde nuestra perspectiva, una colección paleontológica en México puede beneficiarse del continuo curatorial, esquema que reúne los procedimientos básicos de curación organizados en grados, adaptados a las características propias de una colección paleontológica (Hughes et al., 2000; Tabla 2). El continuo curatorial podría ser utilizado como un estándar para establecer procedimientos de curación, como guía para presupuestar los recursos monetarios necesarios, o como auxiliar en la planeación y obtención de la infraestructura apropiada. Por otra parte, el continuo puede utilizarse como un mecanismo de evaluación en la eficiencia de los procedimientos curatoriales, y de esta manera obtener un índice de calidad.

La evaluación efectuada en la Colección de Tipos ( $\sim 9000$ ejemplares) perteneciente a la Colección Nacional de Paleontología, reveló que la calidad de la curación se ubica entre los grados 4 y 5 del continuo (Tabla 2, Figura 3). Debido a que una colección sin soporte curatorial rápidamente se deteriora y la información se pierde, el mantener una pequeña colección en los grados 4 y 5 tiene implícito una fuerte inversión de dinero, esfuerzo y tiempo, pero con ello la Colección Nacional de Paleontología cumple su función como colección científica, su misión institucional y sus obligaciones legales.

Los últimos aspectos no son triviales, lo que merece ahondar en el tema. La institución que alberga a la Colección Nacional de Paleontología es el Instituto de Geología de la Universidad Nacional Autónoma de México. Una de sus funciones de acuerdo a como está establecido su reglamento interno es el "participar activamente en el resguardo científico y cultural de los bienes geológico-paleontológicos de la Nación". Con ello el Instituto requiere y reconoce su responsabilidad para albergar y garantizar la existencia perpetua de la colección paleontológica.

Por otra parte, la Colección Nacional y el resto de colecciones paleontológicas en México están protegidas

Tabla 2. Continuo curatorial de Hughes et al. (2000).

Actividad curatorial para colecciones paleontológicas

Grado 1

Adquisición de ejemplares

Ingreso formal a la colección
Grado 2

Ejemplares clasificados por localidad

Descripción de los ejemplares ingresados

Difusión parcial de la información a la comunidad científica
Grado 2 completo, además de

Ejemplares clasificados por principales grupos taxonómicos o características geológicas

Datos de localidad registrados y capturados en una base de datos electrónica

Datos de localidad asociados a ejemplares por medio de marcas preeliminares o números de registro institucional

\begin{tabular}{|c|c|}
\hline Grado 4 & Grado 5 \\
\hline Grado 3 completo, además de & Grado 4 completo, además de \\
\hline $\begin{array}{l}\text { Taxones identificados o problemas } \\
\text { resueltos (p. ej. biofacies) }\end{array}$ & Lotes de taxones o localidad catalogados \\
\hline $\begin{array}{l}\text { Ejemplares clasificados por grupo } \\
\text { taxómico o asociaciones }\end{array}$ & Ejemplares marcados con números de catálogo \\
\hline \multirow[t]{2}{*}{ Ejemplares bien preparados } & Ejemplares etiquetados \\
\hline & $\begin{array}{l}\text { Lotes acomodados de acuerdo a un marco } \\
\text { taxonómico o geológico }\end{array}$ \\
\hline Ejemplares en contenedores & $\begin{array}{l}\text { Datos del lote (incluyendo historia de } \\
\text { adquisición y localidad) capturados en una } \\
\text { base de datos electrónica }\end{array}$ \\
\hline
\end{tabular}




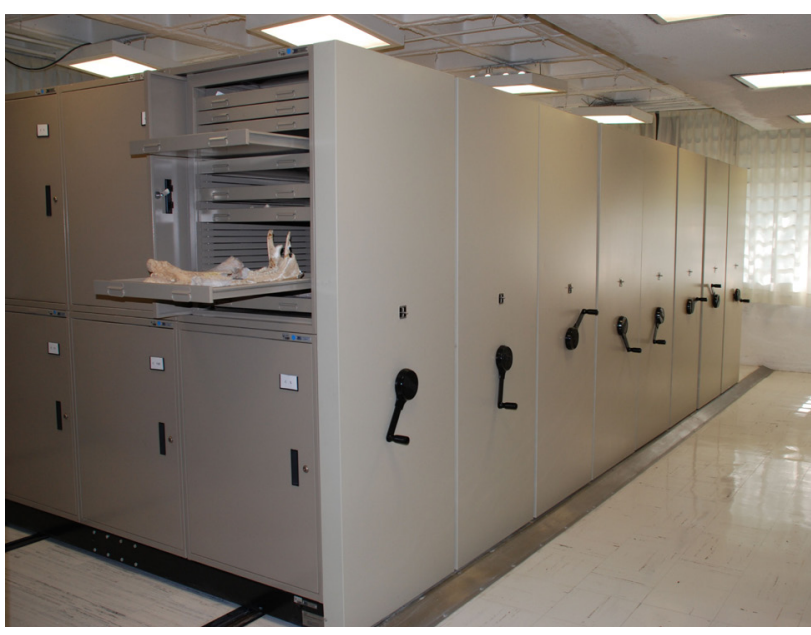

Figura 3. Resguardo de la Colección de Tipos, Colección Nacional de Paleontología del Instituto de Geología de la UNAM. Esta subcolección es quizás la parte más importante del acervo. En ella se encuentran los ejemplares hallados en el territorio nacional que han sido descritos en artículos de revistas arbitradas. Parte de su calidad de curación se manifiesta en las condiciones del resguardo: gabinetes de acero al carbono laminado totalmente limpios, ordenados sistemáticamente, montados en anaqueles móviles, en condiciones de humedad relativa controlada (HR: $\sim 50 \%$ ). Cada gabinete contiene una serie de gavetas deslizables y desmontables, las cuales soportan el peso de numerosos ejemplares de distinta naturaleza. Dicha infraestructura y condiciones ambientales protegen de manera significativa a los ejemplares. Fotografía: J. M. Contreras.

por un amplio marco jurídico, conformado por los artículos constitucionales 27 y 73, por la Ley General de Bienes Nacionales y la Ley Federal sobre Monumentos y Zonas Arqueológicos, Artísticos e Históricos.

Con base en el Artículo 27 párrafos primero, cuarto, quinto y sexto, en los cuales se establece como prioridad de la Nación a los recursos naturales; y al Artículo 73, el cual otorga al Congreso de la Unión la facultad para legislar sobre el registro fósil; se promulgó la Ley General de Bienes Nacionales el 20 de mayo de 2004. En su Artículo 3 y 6 Fracción XVIII considera como bienes de la Nación a régimen de dominio público a "[...] las piezas etnológicas y paleontológicas; los especímenes tipo de la flora y de la fauna; las colecciones científicas o técnicas [...]."

Por otra parte, la reforma a la Ley Federal sobre Monumentos y Zonas Arqueológicos, Artísticos e Históricos, publicada en el Diario Oficial de la Federación el 13 de enero de 1986, homologa el registro fósil con el registro arqueológico. El Artículo 28bis dice: "Para los efectos de esta Ley y de su Reglamento, las disposiciones sobre monumentos y zonas arqueológicos serán aplicables a los vestigios o restos fósiles de seres orgánicos que habitaron el territorio nacional en épocas pretéritas y cuya investigación, conservación, restauración, recuperación o utilización revistan interés paleontológico, circunstancia que deberá consignarse en la respectiva declaratoria que expedirá el Presidente de la República".

Así y de acuerdo con los Artículos 21, 22, 29, 30, 31 y 44, los fósiles hallados en el territorio nacional son propiedad de la Nación, por lo que su venta es ilegal; la recolecta requiere de un permiso otorgado por el Instituto Nacional de Antropología e Historia y toda colección paleontológica pública o privada, deberá estar inscrita en el Registro Público de Monumentos y Zonas Arqueológicos. En el registro se adquiere la responsabilidad legal de la custodia y protección de los fósiles.

Usando como ejemplo a la Colección Nacional de Paleontología se ha mostrado las ventajas de la institucionalización y la definición de una misión, la elaboración de normas y reglamentos de operación y administración, y se ha presentado el conocimiento del marco jurídico. Todos estos aspectos son factores indispensables para la protección de las colecciones paleontológicas, sobre todo cuando desafortunadamente existen prácticas que atentan contra la conservación del registro fósil, contra las colecciones y contra la paleontología misma. Entre las más recurrentes están:

1. Los intentos de formar colecciones públicas o privadas que pretenden ser de tipo científico e incluso sin el conocimiento, el personal, los recursos ni la infraestructura apropiada;

2. Opuesto a lo anterior, la falta de respaldo institucional y apoyo de la comunidad científica para la creación de colecciones cuando se tienen las condiciones necesarias;

3. La falta de compromiso del personal responsable y la carencia de personal capacitado;

4. El uso de las colecciones como mecanismos para la obtención de beneficios personales o de grupo, en términos económicos, académicos, políticos, etc.;

5. El uso privado o selectivo de colecciones públicas; $\mathrm{y}$

6. Falta de voluntad de la comunidad científica para entender y respetar reglamentos y normas operativas.

Éstas y otras prácticas son manifestaciones de un problema de difícil tratamiento y solución al corto plazo, pero que en nuestra opinión es catalizado por el desconocimiento que existe sobre la naturaleza, función, e importancia de las colecciones científicas. Esta carencia junto con la falta de difusión sobre la naturaleza de colecciones paleontológicas fueron los principales motivos detrás del presente artículo, sin embargo éste no puede combatir la falta de interés y la inacción.

\section{Conclusión}

Las colecciones científicas no son simples gabinetes empolvados con objetos inútiles en su interior ni entidades estáticas. Por el contrario, las colecciones científicas son entidades dinámicas directamente relacionadas con la generación y validación del conocimiento científico y cuyo acervo está constituido por los bienes de la Nación, entre éstos los recursos naturales que generan riqueza material y 
otros beneficios sociales. Las colecciones paleontológicas tienen acervos constituidos principalmente por fósiles, conservados en perpetuidad por medio de la curación, pues constituyen la materia prima de investigación científica, garantes de la existencia de la paleontología mexicana. Muchos descubrimientos científicos se han hecho en las colecciones científicas, y no hay mejor ejemplo para colecciones paleontológicas que los fósiles de Burgess Shale (Gould, 1989). ¿Cuántos descubrimientos esperan en nuestras colecciones científicas? La ignorancia o falta de interés catalizan la proliferación de prácticas irresponsables alrededor de las colecciones, mismas que atentan no solo contra éstas, sino también contra el desarrollo del país.

\section{Referencias}

Allmon, W.D., 1994, The value of natural history collections: Curator, 37, 83-89.

Allmon, W.D., 2000, Collections in paleontology, en Lane, R.H., Steininger, F.F., Kaesler, R.L., Ziegler, W., Lipps, J. (eds.), Fossils and the future Paleontology in the $21^{\text {st }}$ century: Frankfurt am Main, Alemania, Senckenberg-Buch, 74, 203-214.

Allmon, W.D., 2005, The importance of museum collections in paleobiology: Paleobiology, 31, 1-5.

Brunton, C.H.C., Besterman, T.P., Cooper, J.A. (eds.), 1985, Guidelines for the curation of geological materials: Londres, The Geological Society, Miscellaneous Paper 17, 214 p.

Clark, J.M., Hopson J.A., Hernández R., Fastovsky D.E., Montellano M., 1998, Foot posture in a primitive pterosaur, Nature, 391, 886-889.

Cotterill, F.P.D., 1997, The second Alexandrian tragedy and the fundamental relationship between biological collections and scientific knowledge, en Nudds, J.R., Pettitt, C.W. (eds.), The value and valuations of natural science collections international Conference, 1995: Manchester, Reino Unido, The Geological Society, 227-241.

Cranbrook, Earl of, 1997, The scientific value of collections, en Nudds, J.R., Pettitt, C.W. (eds.), The value and valuations of natural science collections international conference, 1995: Manchester, Reino Unido, The Geological Society, 3-10.

Dalton, R., 2003, Natural History Collections in crisis as funding is slashed: Nature, 423, 575.

Fitzgerald, G.R., Whiting, P., Shepher, K., 1997, A comparison of methodologies used for valuation of the fish collection at the Canadian Museum of Nature, en Nudds, J.R., Pettitt, C.W. (eds.), The value and valuations of natural science collections international conference, 1995, Manchester, The Geological Society, 110-116.

Gánem, C.E., 2003, Divulgación de la ciencia y progreso social: Ciencia y Desarrollo, 29, 23-24.

Gould, S.J., 1989, Wonderful life: The Burgess Shale and the nature of history: New York, W.W. Norton, 347 p.

Gowland, R.G., 1997, Valuations: a professional's view, en Nudds, J.R., Pettitt, C.W. (eds.), The value and valuations of natural science collections international conference, 1995: Manchester, Reino Unido, The Geological Society, 187-190.
Hughes, N.C., Collier, F.J., Kluessendorf, J., Lipps, J., Taylor, W.L., White, R.D., 2000, Fossil invertebrate and microfossil collections: Kinds, uses and users, en White, R.D., Allmon, W.D. (eds.), Guidelines of the management and curation of invertebrate fossil collections: Boulder, Colorado, E.U.A., The Paleontological Society Special Publications 10, 25-35.

International Council of Museums (ICOM). 2007a. Statuts du Conseil International des Musées (ICOM). Adoptés par la 22e Assemblée générale tenue à Vienne (Autriche), le 24 août 2007 (en línea): disponible en: $<$ http://icom.museum/who-we-are/the-organisation/ icom-statutes/3-definition-of-terms/L/0.html.html >, consultado 13 de junio de 2011.

International Council of Museums (ICOM). 2007b. Development of the museum definition according to ICOM Statutes (2007-1946) (en línea), disponible en: $<$ http://archives.icom.museum/hist_def_eng. html $>$, consultado 13 de junio de 2011.

Khun, T.S., 1962, The structure of scientific revolutions: Chicago, University of Chicago Press, $173 \mathrm{p}$.

Krikken, J., 1997, A Dutch exercise in the valuation of natural history collections, en Nudds, J.R., Pettitt, C.W. (eds.), The value and valuations of natural science collections international conference, 1995: Manchester, Reino Unido, The Geological Society, 124-126.

Miller, B., Conway, W., Reading, R.P., Wemmer, C., Wildt, D., Kleiman, D., Monfort, S., Rabinowitz, A., Armstrong, B., Hutchins, M., 2004, Evaluating the conservation mission of zoos, aquariums, botanical gardens and natural history museums: Conservation Biology, 18, 86-93.

National Science and Technology Council, Committee on Science, Interagency Working Group on Scientific Collections, 2009, Scientific Collections: Mission-critical infrastructure of Federal Science Agencies: Washington, D.C., Office of Science and Technology Policy, $49 \mathrm{p}$.

Nudds, J.R., Pettitt, C.W. (eds.), 1997, The value and valuations of natural science collections international conference, 1995: Manchester, Reino Unido, The Geological Society, 276 p.

Stansfield, G., 1994, Functions and organization of natural history museums, en Stansfield, G., Mathias, J., Reid, G. (eds.), Manual of Natural History Collections curatorship, Museums and Galleries Commission: Londres, Her Majesty Storage Office, 1-10.

Suarez, A.V., Tsutsui, N.D., 2004, The value of museum collections for research and society: Bioscience, 54, 66-74.

Tate, A., Skinner, T., 1992, Storage systems, en Thompson, J.M.A. (ed.), Manual of curatorship. A guide to museum practice: Oxford, Reino Unido, Butterworth-Heinemann, 459-467.

Tobin, M.F. y Evans, T., 2004. Collective knowledge: The value of natural sciences collections (en línea): Washington, D.C., Natural Science Collection Alliance, disponible en $<\mathrm{http}$ ://nscalliance.org/?page id $=10>$, consultado 13 de junio de 2011.

Uribe, F., 1997, The evaluation of natural history collections: some remarks, en Nudds, J.R., Pettitt, C.W. (eds.), 1997, The value and valuations of natural science collections international conference, 1995: Manchester, Reino Unido, The Geological Society, 1581-162.

Manuscrito recibido: Junio 1, 2011.

Manuscrito corregido recibido: Julio 10, 2011.

Manuscrito aceptado: Julio 12, 2011. 